Ebisu

Études japonaises

Études japonaises

47 | printemps-été 2012

Catastrophes du 11 mars 2011, désastre de

Fukushima : fractures et émergences

\title{
« Un paradis habité par des meurtriers sans méchanceté et des victimes sans haine »: Hiroshima, Tchernobyl, Fukushima
}

"A Paradise Inhabited by Murderers Deprived of Wickedness and Victims

Deprived of Hatred": Hiroshima, Tchernobyl, Fukushima

「悪意無殺害者たちと増悪無犠牲者たちの住む天国」一広島、チェル ノブイリ、福島

Jean-Pierre Dupuy

\section{OpenEdition}

Journals

Édition électronique

URL : http://journals.openedition.org/ebisu/245

DOI : 10.4000/ebisu.245

ISSN : 2189-1893

Éditeur :

Institut français de recherche sur le Japon (UMIFRE 19 MAEE-CNRS), Maison franco-japonaise

Édition imprimée

Date de publication : 1 juin 2012

Pagination : 49-57

ISSN : 1340-3656

Référence électronique

Jean-Pierre Dupuy, « "Un paradis habité par des meurtriers sans méchanceté et des victimes sans haine » : Hiroshima, Tchernobyl, Fukushima », Ebisu [En ligne], 47 | printemps-été 2012, mis en ligne le 03 avril 2014, consulté le 22 avril 2019. URL : http://journals.openedition.org/ebisu/245 ; DOI : 10.4000/ebisu.245 


\section{«Un paradis habité par des meurtriers sans méchanceté et des victimes sans haine » Hiroshima, Tchernobyl, Fukushima*}

Jean-Pierre DUPUY

\section{Un nouveau régime du mal}

En 1958, le philosophe allemand Günther Anders se rendit à Hiroshima et à Nagasaki pour participer au $4^{\mathrm{e}}$ Congrès international contre les bombes atomiques et les bombes à hydrogène. Il tint pendant tout ce temps un journal. Après de nombreux échanges avec les survivants de la catastrophe, il note ceci :

« La constance qu'ils mettent à ne pas parler des coupables, à taire que l'événement a été causé par des hommes ; à ne pas nourrir le moindre ressentiment, bien qu'ils aient été les victimes du plus grand des crimes - c'en est trop pour moi, cela passe l'entendement. "

Et il ajoute :

" De la catastrophe, ils parlent constamment comme d'un tremblement de terre, comme d'un astéroïde ou d'un tsunami."

Professeur émérite de philosophie politique à l'École polytechnique, Paris. Professeur de sciences politiques à l'université Stanford. Membre de l'Académie des Technologies. Président du Comité d'éthique et de déontologie de l'Institut français de radioprotection et de sécurité nucléaire.

* Ce texte est tiré d'une conférence donnée à l'université de Tokyo, le 30 juin 2011. 
À peu près en même temps que Hannah Arendt, sa condisciple, qui fut aussi sa femme, Anders tentait d'identifier un nouveau régime du mal. Arendt parlait d'Auschwitz, Anders d'Hiroshima. Arendt avait diagnostiqué l'infirmité psychologique d'Eichmann comme « manque d'imagination ". Anders montrait que ce n'est pas l'infirmité d'un homme en particulier, c'est celle de tous les hommes lorsque leur capacité de faire, qui inclut leur capacité de détruire, devient disproportionnée à la condition humaine. Alors le mal s'autonomise par rapport aux intentions de ceux qui le commettent. Anders et Arendt pointaient ce scandale qu'un mal immense peut être causé par une absence complète de malignité ; qu'une responsabilité monstrueuse puisse aller de pair avec une absence totale de méchanceté. Nos catégories morales sont impuissantes à décrire et juger le mal lorsqu'il dépasse l'inconcevable. Il faut se résoudre alors à dire, comme Hannah Arendt citant le juriste Yosal Rogat, qu' « un grand crime est une offense contre la nature, de sorte que la terre elle-même crie vengeance ; que le mal viole l'harmonie naturelle que seul le châtiment peut rétablir " (Arendt 2002 : 478-479). Le fait que les juifs d'Europe aient substitué au mot " holocauste " celui de shoah, qui signifie catastrophe naturelle, et, singulièrement, raz de marée, tsunami, atteste cette tentation de naturaliser le mal lorsque les hommes deviennent incapables de penser cela même dont ils sont victimes ou responsables.

Hiroshima, comme Auschwitz, un tsunami ? Quelle ironie du sort : à Fukushima, c'est un véritable tsunami, une onde on ne peut plus matérielle, qui est venue réveiller le tigre nucléaire. Certes, il s'agit d'un tigre en cage : un réacteur électronucléaire n'est pas une bombe atomique. Il en est en un sens la négation puisqu'il consiste à brider une réaction en chaîne qu'il a lui même provoquée. Cependant, dans le domaine symbolique, la dénégation affirme cela même qu'elle nie. Lorsque nous disons : "Fukushima n'a rien à voir avec Hiroshima ", nous faisons explicitement le rapprochement que nous disons ne pas vouloir faire. De plus, dans la réalité, et nous y sommes, il arrive que le tigre s'échappe de sa cage.

Mais Fukushima, c'est aussi une catastrophe industrielle et technologique. L'Occident a surtout retenu cette dimension car c'est la survie même de son modèle de développement économique qui est en jeu. Pris en tenaille entre le réchauffement climatique et l'épuisement des ressources fossiles, il espérait que l'énergie nucléaire serait sa voie de salut. Il se peut que Fukushima sonne le glas de cet espoir. 
La perte des différences n'est donc pas seulement entre Hiroshima, catastrophe morale, et le tsunami, catastrophe naturelle, elle est aussi entre Hiroshima, catastrophe morale, et Fukushima, catastrophe industrielle et technologique. Dans le premier cas, Hiroshima, le mal résulte de l'intention de commettre le mal ; dans le second, Fukushima, le mal résulte de l'intention de commettre le bien. Le grand critique de la société industrielle que fut Ivan Illich (1926-2002) appelait contreproductivité ce retournement tragique. Il affirmait que les plus grandes menaces viennent aujourd'hui moins des méchants que des industriels du bien. On doit moins redouter les mauvaises intentions que les entreprises qui, comme l'Agence internationale pour l'énergie atomique, se donnent pour mission d'assurer « la paix, la santé et la prospérité dans le monde entier ». Les antinucléaires qui se croient tenus pour mener leur combat de dépeindre leurs ennemis de la façon la plus noire ne comprennent pas qu'ils affaiblissent ainsi leur critique. Il est beaucoup plus grave que les opérateurs des mégamachines qui nous menacent soient des gens compétents et honnêtes. Ils ne peuvent comprendre qu'on s'en prenne à eux.

Cette autonomisation du mal par rapport aux intentions de ceux qui le commettent est le thème principal de ma réflexion sur le mal. J'ai donné comme titre à ce texte une citation extraordinaire de Günther Anders, qui apporte une bonne nouvelle apparemment, puisqu' elle annonce la « fin de la haine ". Cette fin de la haine n'est cependant pas la bonne nouvelle de l'Evangile chrétien, le message d'amour, c'en est même tout le contraire. Anders écrit en effet :

"À l'instant même où le monde devient apocalyptique, et ce par notre faute, il offre l'image ... d'un paradis habité par des meurtriers sans méchanceté et des victimes sans haine. Nulle part il n'est trace de méchanceté, il n'y a que des décombres. "

\section{La leçon de Tchernobyl}

Je veux prendre comme illustration de mon propos sur le nouveau régime du mal et sa déconnexion par rapport aux intentions des agents humains, la question très controversée des effets d'une longue exposition d'une importante population à une faible radioactivité. Cette question a empoisonné le débat sur les conséquences en termes de mortalité et de morbidité de 
l'accident de Tchernobyl et elle est en train d'être reprise à nouveaux frais à l'occasion de la catastrophe de Fukushima.

C'est essentiellement à cause de désaccords fondamentaux sur la question des " faibles doses » que des valeurs aussi fantastiquement différentes que 40 et 400000 morts provoquées par Tchernobyl ont pu être avancées avec la même bonne foi par des évaluateurs différents. Où donc est la vérité objective, scientifique, en la matière?

Poser la question en ces termes, c'est présupposer qu'il existe une norme d'évaluation scientifique séparable d'autres normes, de nature éthique ou juridique, par exemple. Je soutiens que, dans le cas considéré au moins, il n'en est rien.

Pour le montrer, il suffit d'analyser les conclusions du Forum Tchernobyl, l'organisme international et onusien qui a été constitué pour tirer les leçons de la catastrophe du 26 août 1986'. La centaine d'experts qui ont travaillé pour ce Forum, des physiciens, des biologistes, des médecins, des économistes, sont unanimement arrivés à des conclusions dont ils sont persuadés qu'elles constituent le dernier mot de la vérité scientifique. Ils admettent, certes, qu'il existe d'autres vérités, qu'ils appellent " humaines ", "sociologiques » ou " psychologiques ». Cette concession immunise l'objectivité scientifique revendiquée comme valeur de toute contamination par ce qui n'est pas elle. Or, dans l'évaluation des conséquences d'une catastrophe en général, de celle de Tchernobyl en particulier, il est douteux qu'il existe un domaine séparé qui serait celui de l'évaluation scientifique. Cette expression sonne d'ailleurs comme une contradiction dans les termes: dans le domaine des valeurs, la science seule n'a rien à dire.

En 2001, un expert pouvait dire :

"Tchernobyl a causé trente et une morts, suite aux lésions causées par deux cents sieverts, cliniquement attribués à l'exposition aux radiations, ainsi que deux mille cancers

1. À la demande de l'Agence internationale de l'énergie atomique (AIEA), le Forum Tchernobyl a été mis sur pied en 2002 pour évaluer les conséquences de la catastrophe. Il regroupait huit organisations internationales dépendant de l'ONU, parmi lesquelles l'Organisation mondiale de la santé (OMS), le Programme des Nations unies pour l'environnement, la Banque mondiale, le Comité scientifique des Nations unies pour l'étude des effets des rayonnements ionisants (UNSCEAR) et l'AIEA elle-même, ainsi que les gouvernements de la Russie, de l'Ukraine et de la Biélorussie. 
aisément curables de la thyroïde chez l'enfant. À ce jour, il n'existe aucune preuve, validée internationalement, d'un impact sur la santé publique qui soit attribuable à Tchernobyl par exposition - je souligne : par exposition aux radiations ${ }^{2}$.»

Cette insistance sur la notion de conséquence causale de l'exposition aux radiations s'explique par le fait que la thèse officielle ne nie pas que la catastrophe ait eu des " conséquences socio-psychologiques » considérables. Ceux-là mêmes qui chiffrent les décès en dizaines et non pas en dizaines de milliers n'hésitent pas à affirmer que Tchernobyl représente la plus grande catastrophe que le nucléaire civil ait jamais connue jusqu'ici. Comment se reconnaître dans le dédale de ces apparentes contradictions?

La dialectique implicite à la thèse officielle se décline en trois temps :

1) La catastrophe de Tchernobyl a produit une radioactivité considérable (des centaines de fois plus de matières radioactives rejetées qu’à Hiroshima), mais les conséquences causales de l'exposition aux radiations auront été infimes par rapport à ce qui a été craint, annoncé, répercuté par des médias intéressés au sensationnel. L’objectivité scientifique commande de l'affirmer avec force.

2) Les populations concernées ont été en vérité très gravement affectées, mais c'est parce qu'elles croyaient avoir été très gravement affectées, dans l'ignorance où elles sont restées du point 1). Par exemple, le stress, l'angoisse ont pu produire les pathologies étranges que l'on observe dans les zones contaminées; ou bien, les habitants attribuent systématiquement les maux qui les accablent à la radioactivité et, vivant celle-ci comme une fatalité, négligent de se soigner.

3) Le troisième moment dérive des deux premiers : pour briser les mécanismes responsables de l'écart entre la vérité objective 1) et les croyances autoréalisatrices 2), il faut informer, communiquer, éduquer de façon que les gens en arrivent à vivre avec la chose, l'apprivoisent, se déprennent de toute angoisse - comme on apprend à vivre sans peur dans l'espace autoroutier en se soumettant à quelques règles simples et finalement peu contraignantes.

2. Propos tenus par le représentant de l'AIEA à la conférence de l'OMS qui s'est tenue à Kiev en juin 2001. 
Pourquoi cette vérité officielle a-t-elle fait et fait-elle encore l'objet de tant de contestations ? Pour le vingtième anniversaire de la catastrophe, en avril 2006, la revue Nature a publié un numéro spécial, dont la conclusion était :

"Si une étude complète et indépendante des conséquences de l'accident nucléaire le plus terrible à l'échelle mondiale n'est pas réalisée, et ses résultats publiés d'une telle façon que tout un chacun puisse en comprendre la teneur et s'en faire une idée juste, des estimations follement divergentes continueront d'être énoncées, et la défiance que le public éprouve à l'endroit de l'industrie nucléaire continuera d'augmenter. "

Cet avertissement n'a pas été entendu et Fukushima a fait déborder le vase, si l'on en croit l'épidémie de renoncements au nucléaire à laquelle on assiste à présent.

L'estimation des effets sur la santé humaine d'une catastrophe nucléaire recourt en principe à trois méthodes : l'observation directe, l'enquête épidémiologique et la modélisation. Les sauveteurs des premières heures ont reçu à Tchernobyl des doses telles que leur mort peut être attribuée en toute certitude à l'accident. Pour toutes les personnes qui ont subi par la suite des doses moyennes ou faibles, les choses sont beaucoup plus complexes. En principe, une enquête épidémiologique pourrait apprécier rétrospectivement l'excès des maladies malignes qui ont affecté les populations touchées sur le taux normalement attendu. Cette enquête n'a pu être faite correctement dans le cas de Tchernobyl, pour deux raisons : d'une part, les populations les plus touchées, c'est-à-dire les liquidateurs et les personnes que l'on a dû déplacer, se sont dispersées sur tout le territoire de l'Union soviétique, et aucun suivi sérieux n'a pu avoir lieu; pour les millions de personnes qui ont reçu de faibles doses, d'autre part, une étude épidémiologique n'aurait pu détecter un éventuel accroissement du taux de mortalité par leucémie ou cancer, de toute évidence faible, voire très faible, qu'au prix de moyens exorbitants que l'Union soviétique au bord de la décomposition ne pouvait mobiliser. C'est donc la modélisation qui s'est substituée à l'enquête épidémiologique, cette même modélisation à laquelle on doit de toute façon avoir recours pour estimer les morts à venir.

Le modèle retenu par les autorités internationales de radioprotection a été un modèle " linéaire sans seuil " : il est donc supposé que l'effet sur la morbidité et la mortalité est proportionnel à la dose reçue même pour les 
très faibles doses. En d'autres termes, il n'existe aucun seuil de radiations en deçà duquel l'effet est postulé être nul.

Cependant, lorsqu'on lit le rapport du Forum Tchernobyl avec attention, on découvre que le chiffre de 4000 morts annoncées comme étant le bilan définitif n'a été calculé au moyen du modèle linéaire sans seuil que sur une petite partie de la population mondiale que les radiations ont touchée : tout juste 600000 personnes, soit environ 200000 liquidateurs, 120000 personnes évacuées et 270000 autres résidant dans les zones les plus contaminées. Quant aux millions de personnes autres touchées, l'estimation officielle ne se prononce pas à leur sujet, ce que beaucoup ont compris comme signifiant que la catastrophe n'était responsable d'aucune mort parmi elles. Si on leur applique à elles aussi, ne serait-ce que pour des raisons de cohérence interne, le modèle linéaire sans seuil, on trouve un nombre de morts égal à quelques dizaines de milliers, et non pas quelques milliers.

Que s'est-il passé ? Lorsque les doses radioactives sont très étalées dans le temps et distribuées sur une vaste population, il est impossible de dire d'une quelconque personne désignée qui meurt d'un cancer ou d'une leucémie qu'elle est morte du fait de Tchernobyl. Tout ce que l'on peut dire, c'est que la probabilité qu'elle avait a priori de mourir d'un cancer ou d'une leucémie a été très légèrement accrue du fait de Tchernobyl. Les trente ou quarante mille morts qu'aura causées la catastrophe nucléaire selon le modèle linéaire sans seuil ne peuvent donc être nommées. La thèse officielle consiste à en conclure qu'elles n'existent pas. Il y a là une subtilisation d'une forêt de cadavres d'un genre très particulier. C'est en ce point précis que l'évaluation scientifique se révèle inséparable d'une prise de position éthique et même métaphysique.

Le problème philosophique profond qui se cache derrière cette discussion est explicitement traité dans l'un des ouvrages les plus importants et les plus influents de la philosophie morale du vingtième siècle, Reasons and Persons, du philosophe britannique Derek Parfit (1984). Ce livre fut publié en 1984, deux ans avant la catastrophe. Sous le nom de "Cinq erreurs majeures en mathématiques morales ", il démonte de façon prémonitoire la série de raisonnements auxquels se sont livré les experts en catastrophe nucléaire ou en radioprotection. 
Parmi les cinq erreurs analysées par Parfit, j'isole les deux suivantes. Il existe des actions ou des faits qui ont une probabilité extrêmement faible de produire un effet considérable. Parce qu'elles sont insignifiantes, un calcul moral ou rationnel peut être tenté de tenir ces probabilités pour nulles. Il existe des actions ou des faits qui produisent des effets imperceptibles mais qui touchent un très grand nombre de personnes. Parce que ces effets sont imperceptibles, on a envie de les passer par pertes et profits. C'est, dans l'un et l'autre cas, tomber dans le piège du paradoxe du sorite (littéralement : le paradoxe du tas), connu depuis le Iv ${ }^{\mathrm{e}}$ siècle avant Jésus-Christ : un cheveu greffé sur le crâne d'un chauve ne le transforme pas en non-chauve ; et cependant un non-chauve est une personne qui a un certain nombre de cheveux.

Le paradoxe du sorite n'est pas une invention de philosophe désœuvré, nous le rencontrons chaque fois que nous votons dans une élection nationale qui oppose deux candidats. Sauf dans le cas extrêmement improbable (peut-être une chance sur un milliard) où les suffrages se répartiraient également entre les deux options, il est incontestable que le bulletin déposé dans l'urne par chacun des électeurs aura eu un effet nul. À la question : "Le résultat final eût-il été changé si j'avais voté autrement que je l'ai fait (ou bien si je n'avais pas voté) ? ", chacun doit répondre : non ! Et cependant, le résultat du vote découle immédiatement du décompte des voix. Or nous savons résoudre ce paradoxe. Il suffit de recourir au mode de pensée symbolique, ce que, dans ces situations, nous faisons spontanément. Nous interprétons les résultats de tels votes, même ou surtout lorsqu'ils sont serrés, comme la manifestation du choix soigneusement délibéré d'un sujet collectif : le peuple, l'électorat, etc. Du point de vue d'une conception étroite de la rationalité, ce sujet collectif qu'on appelle en renfort est une pure fiction. Il dissout cependant le paradoxe au plan moral, qui est ici celui de la responsabilité.

En 1991, la consultation concernant le traité de Maastricht qui gouverne l'Union européenne a donné en France l'avantage au oui, mais d'extrême justesse. On a dit : "Dans sa grande sagesse, le peuple français a répondu oui à l'Europe, mais il a aussi voulu donner un avertissement à tous ceux qui voulaient précipiter les événements ". Si cette manière de parler est légitime, qui fait d'un sujet fictif le porteur d'une délibération et le responsable d'un état de choses, celle qui affirme : "La catastrophe de Tchernobyl est responsable de dizaines de milliers de morts ", ne l'est pas moins. Le 
paradoxe du sorite est le même dans le cas du vote et dans celui des effets sur la santé de faibles doses de radioactivité.

On voit en tout cas sur cet exemple que l'évaluation objective ne peut faire l'économie d'une prise de position d'ordre moral. Les morts de Tchernobyl sont irrémédiablement des morts statistiques, virtuels, leur nombre ne peut résulter d'une énumération, seulement d'un calcul. Décider de ne pas les compter est un choix éthique. Faire le choix inverse ne l'est pas moins.

Que peut-on reprocher aux responsables internationaux de l'énergie atomique? Je fais le pari que ce sont des gens compétents et honnêtes.

J'ai fait cette hypothèse dans mon livre Retour de Tchernobyl. Journal d'un homme en colère (2006) et cela m'a valu des menaces de mort de la part des militants anti-nucléaires de mon pays. Ils n'ont pas compris que cette hypothèse est celle qui maximise la gravité de notre situation présente. Les intentions de ceux qui nous gouvernent n'ont pas d'importance. C'est la situation qui en a. Pour être un responsable aujourd'hui du nucléaire civil, il faut avoir cette forme d'esprit qui conduit à reléguer les questions éthiques dans un domaine séparé et, finalement, secondaire. C'est là la source principale du mal, et non les intentions supposément malveillantes des opérateurs du nucléaire.

\section{Bibliographie}

ARENDT Hannah, 2002

Eichmann à Jérusalem, Paris,

Folio, Gallimard.

DUPUY Jean-Pierre, 2006

Retour de Tchernobyl. Journal

d'un homme en colère, Paris, Seuil.
PARFIT Derek, 1984

Reasons and Persons, Oxford,

Clarendon Press. 